\title{
The Development Mechanisms Investigation of Ultrafast Processes in the Economy
}

\author{
Margarita Alekseevna Afonasova ${ }^{1}$ \\ ${ }^{1}$ Department of Economy, Tomsk State University of Systems Management and Radio Electronics, Tomsk, \\ Russia \\ Correspondence: Margarita Alekseevna Afonasova, Department of Economy, Tomsk State University of Systems \\ Management and Radio Electronics, Lenina st., 40, Tomsk, 634050, Russia. E-mail: afonasova@yandex.ru
}

\author{
Received: October 14, 2014 Accepted: November 11, 2014 Online Published: November 15, 2014 \\ doi:10.5539/res.v6n4p216 \\ URL: http://dx.doi.org/10.5539/res.v6n4p216
}

\begin{abstract}
The article is devoted to the research of the mechanisms of acceleration and impeding of the economy in the period of unstable states of the society, intensification of the threats of the global economic recession for detection of the preconditions of development of the super-speed processes in the economic systems, appearing in the periods of non-stability. The new interpretation of the essence and content of the super-speed processes in the economy, the role of the positive and negative inverse connections, as well as the hypothesis about possibility of formation of the model of the accelerated growth of the economy on the basis of the usage of the potential of the mechanisms of the super-speed processes were stated in the article. The mechanisms of impeding were considered and the factors causing the effect of acceleration were detected, the attempt was made to explain the nature of the mechanisms of development of the super-speed processes in the economy, as well as to detect the means and instruments of supporting the stability of the economic system in the period of crisis.
\end{abstract}

Keywords: development, economics, ultrafast processes, feedbacks, factors crisis, sustainability, management

\section{Introduction}

Increasing globalization, economic, scientific and technical integration, unsteady social conditions destabilize the development of national economies, contribute to the emergence of ultrafast processes development modes, when certain parameters (values) increase unrestrictedly in the finite amount of time. Without revealing the development mechanisms of ultra-speed processes in the economy, as well as tools to sustain economic systems during crises, the threat can not eliminated because under the influence of a new crisis wave they will be strongly deformed and will not return to a stable position.

In this regard, this paper addresses to the following issues:

- Specification of the nature and preconditions of the "ultrafast processes" development in the economy;

- Revealing the role of positive and negative feedbacks in the development of ultrafast processes in economic systems;

- Revealing and characterization of the economy acceleration and deceleration mechanisms in the crisis time;

- Justification of the hypothesis that the ultrafast processes development in the economy is influenced by both accelerating and braking factors, which together determine the complex dynamics of modern economic systems.

In the past decades socio-economic development has been taking place in the context of growing complexity of processes and phenomena that generates the nonlinear dynamics of the economy and society. It means that the elements and processes develop in the unstable regimes, and the threat of the rapid expansion crisis and its spread to the national and the international community becomes more real.

At the present time many groups of native and foreign researchers, including the Belgian scientific school under Prigogine's supervision developed the non-equilibrium thermodynamics theory, focus on studying the problems of unstable, rapidly developing world.

V. G. Afanasev, G. B. Kleyner, J. Kornai, V. I. Kostyuk, V. V. Mogilevskiy, M. I. Setrov researched functioning and development patterns of socio-economic systems in their works. Over the last years according to the 
interdisciplinary approaches the problems of socio-economic systems were discovered in the works written by S. P. Kurdyumov, E. N. Knyazeva, G. G. Malinetsky, N. N. Moiseev and other authors.

Despite the significant number of scientific papers in the research field of the complex systems behavior in periods of instability and insecurity, the scientific literature does not adequately reflect issues related to the analysis of ultrafast processes development mechanisms in socio-economic systems, that is why the further research is required.

Researchers of the economic dynamics are more and more often concentrating on its analysis from the standpoint of economic evolution and self-organization theories, because the traditional economic theory can no longer cope with the increasing complexity and dynamism of the processes and new challenges of globalization.

In the process of the economic systems evolution the complication of their structure mainly takes place in order to adapt to their existence. Researchers have concluded that the systems of higher complexity are more stable and viable, they take up all the available space and exist until the causative conditions will not have change dramatically (Bransky, 2004).

Currently, there is an understanding the evolutionary processes can be studied only due to the interdisciplinary approach, with a special role given to physics, without the contribution of which, as G. Nikolis and I. Prigogine repeatedly noticed, it is impossible to comprehend the true sense of the evolutionary processes (Nicolis, 1979). The synergetics and the self-organization theory examine the driving forces of the complex systems evolution, as well as making it possible to describe the evolutionary processes dynamics in economic systems by means of the equations systems and models.

The synergetics imparts an understanding of the development processes, different from the dominant way of the world outlook that prevailed during the previous centuries in the classical science. It is based on the ideas of systematicity, non-linearity of development and the interrelation of the chaos and the order (chance and necessity).

The self-organization is a process of spontaneous structure formation, which is the opposite of general tendency towards the structures demolition. The self-organization processes are those processes that lead a system from the equilibrium to the formation of more sophisticated and organized structures by means of the driving forces, inherent to the system itself (Ebeling, 2005).

The evolution in economic systems can be considered as an endless chain of self-organization processes, i.e. as a spiral of self-organization cycles. The relatively stable state of the system sooner or later becomes unstable because of changes in internal or external conditions. The fluctuation initiates the self-organization process that generates new structures. The self-organization causes the emergence of a new relatively steady condition, which can become the beginning of a new cycle.

By analogy with other systems, the evolution of economic systems moves by fits and starts. Sufficiently long stages of soft development alternate with short-term stages of explosive development (disasters). In the evolutionary systems dynamics constructive and destructive processes consistently change each other. However, where the fluctuations weight goes beyond a certain critical boundary, the proportionality is violated, the extent of destruction may exceed the one of creation, and the system transfer into an unpredictable operating mode or crumbling one. Among the decay products of the former structure the evolution rejects the overwhelming majority, and more stable elements are stored and they gain a competitive advantage over the others. Then preserved elements cooperate with each other what usually increases their thermodynamic stability, thus creating a competitive advantage for them.

Consequently, the economic development is an evolutionary process that takes a stochastic manner. It is characterized by the occurrence of periodic dissipation and development of ultrafast processes, when the dynamics is a subject to the principles of "small growths" and "gain fluctuations" at certain stages.

In their studies G. Nicolis and I. Prigogine have repeatedly noted that in nonlinear systems the searching of the stable state plays a role of the natural selection. "A dynamical system that generates chaos acts as a sort of selector, that discards the vast majority of random sequences (bifurcation branches) and stores only those that are compatible with the relevant dynamical laws" (Nicolis, 1990).

Meanwhile, it is known that the selection of preferences towards more sustainable state arrives only when the existing measures of sustainability are violated. That violation of the system stability measures beyond its regeneration capacity causes the bifurcation, which is defined as “... a one-time state when the system having lost stability, completely loses memory, and the next evolution is fundamentally unpredictable, in so far it is determined by only the random factors that act upon the system at the bifurcation point" (Moiseyev, 1999). 
One of two scenarios provides for the choice of the development path: something realizes or discards. This split of the development pathway occurs at the point of the lowest stability which corresponds to the chaos attained. The choice from two (realizable and unrealizable) system evolution scenarios in the synergetics is called bifurcation. The potential trajectory of some structure formation becomes an actual trajectory" (Afonasova, 2010).

The bifurcation presents a branch point of development options. This is a tipping, critical point of the system development where it chooses a path of existing potentially development options. Bifurcations are either soft, when they initially do not differ from the slow evolutionary development, or hard, when the system jumps to a new qualitative state. At the bifurcation points where the system chooses its way, it is particularly sensitive to external influences. In one of these points the strict bifurcation occurs and an upturn for a new development branch takes place.

As a result of the bifurcation, more complex formations with a higher degree of order appear. In general, according to the concept of synergy, the violation of the system stability is only warranted inasmuch as it leads to the stability of a higher order. In this case, the main condition to make up structures of a higher order degree is to exceed the absorption of the negentopy resource over its spending.

The positive origin of destructiveness in the systems development dynamics demonstrates that it is a source to form in the system an order of a higher lever and a push to begin self-organization processes (Afonasova, 2009). The destruction for destruction itself loses an evolutionary sense. Therefore, the great importance in the research of ultrafast processes from the synergetic positions is given to the analysis of cumulative grounds of sustainability, or attractors that transmit the particular system from the unstable state into the new stable state of a higher order. The stability arises from the instability. The formation of more ordered structures involves a chain of successive stability violations in order to make more effective structures (Friedman, 1980).

In all the researches on synergetics the idea that any system is orientated to maintain its sustainability is presented to explain its development mechanisms. The certain area of a steady state of self-organizing system called an attractor serves as an analogue of system forming factor. The attractor structures are similar to evolution targets, as if they draw a whole multitude of system development trajectories. The self-organization of complex dynamic systems, as well as its acquisition of the necessary stability begins in the domain of attraction.

In mathematics the term "attractor" usually denotes some attracting multitude. In synergetics, attractors mean uncertain stable structures in the open nonlinear medium to which all evolutionary processes of this medium come in the end. The attractor essentially draws and subdues all the subsequent events. Wherein the attractor has already constituted, as if we wish to return to the initial phase of the system conditions or to switch to another more favorable attractor, this is unlikely to implement.

While in mathematics, physics and synergetics the subject of attractors is developed fully enough, the role of attractors for the socio-economic systems is researched not so well. The definition of attractor for the socio-economic system should be interpreted differently. In order to formulate the meaning of attractors for society, it is necessary to refer to the specifics of socio-economic systems where causes and effects, both, material and ideal, subjective and objective are interconnected in a complicated way. In conditions of increasing economic risks and emerging social threats, the problem of identification and exploration of attractors for the socio-economic system turns from the merely theoretical area into the actual application problem. To solve this issue it can reach decision how to manage effectively the socio-economic systems transition from the unstable and extreme states to the new quality of their development. Strictly formulated goals, defined normative attitudes and progressive trends together with a vision of the future, virtually presenting in minds of the managing executants, etc. can figure as attractors for the socio-economic systems. Hence, some researchers have concluded that the attractor in the socio-economic system can be represented as a kind of cognitive map of manager's consciousness, where the future state of the managed system had been already recorded.

The emergence of a new attractor in the economic system means a sharp narrowing of the uncertainty scope while choosing an alternative development. The attractor stimulates the system to begin the structural self-organization as a catalyst for intra-system changes.

The economic development process is nonlinear and inhomogeneous. The idea to make possible ultrafast processes development at certain stages, which is based on nonlinear mechanisms of positive feedback, stands behind the nonlinearity. It is known that the negative feedback has a stabilizing effect on the system that "forces" the system itself to come back to the equilibrium. Meanwhile the positive feedback leads to the buildup of the system as well as to its moving away from the equilibrium and transitioning to the instability. The nonlinear positive feedback is an essential element of autocatalytic processes that are currently used both in biological and 
socio-economic systems. An illustrative example from the economics field is a fast, self-stimulating growth of monetary capital invested in speculative transactions on the financial markets under certain conditions for their implementation.

Thus, the nonlinear positive feedback can serve as a source for rapid development, and it provides a mechanism underlying the so-called blow-up regimes. The blow-up regime is a mode of ultrafast processes growth in the open nonlinear media, in which the characteristic values (such as monetary capital) grow without limit in the finite amount of time (Knyazeva, 2005). Blow-up regimes under certain conditions can lead to the localization and the formation of dissipative structures. It should be noted that the blow-up regimes have a long quasi-stationary stage when the effect of small perturbations on the system is irrelevant. Further, when systems have "grown up" and crossed the threshold of slow growth, they can begin to develop in a super-fast pace, in the blow-up regime.

As the examples of such processes in economics we can introduce the rapid growth of the Chinese economy, guaranteed with the full stimulation of investment activity, and the ultrafast process of trade concentration in periods of the speed economic development, when some small stores are displaced with the super- and hypermarkets, as well as some types of speculative exchange transactions. In 2013, Italy initiated the world's first tax on ultrafast exchange transactions. Thresholds for traders were established to change and cancel operations. After this limit each transaction is levied tax of $0.02 \%$ if transactions or their cancellation occur less than a half a second. The other eleven Eurozone countries support these measures.

The Russia still doesn't consider such perspective, although recently the dynamics of ruble rates in relation to dollar and euro bears evident stick-slip nature. Thus, recently in Moscow stock-exchange the non-standard leaps of currency rates: on the 30th October in 2014 the dollar for an hour and a half dropped more than two rubles, and on the trade session on 31st October the dollar strengthened its positions again. On the 6th November in 2014 the rate of dollar on the trades of Moscow stock-exchange has already exceeded 46 RUB, while euro has come beyond the psychologically important border in 58 RUB. Totally since the beginning of year the dollar has risen in price already for $40 \%$.

The economists state that the last leaps were caused by the shock from changes of policy of Centrobank.

It is known that close to the moment of exacerbation in the economic system it becomes possible to increase the microscopic (insignificant) fluctuations and their expansion to macroscopic dimensions. The consequences of this expansion can also be macroscopic and consist of a breach of the pace of economic system development, needed to maintain its integrity and stability. As a result, the probabilistic disintegration of the economic system becomes possible as one of the feasible scenarios to pass instability and the moment of exacerbation.

The analysis made shows when a certain threshold of the information flow growth (with an increase of the information diffusion compared with its production) is achieved, the development law also changes qualitatively (Malinetsky, 2006). The course of the economic development can accelerate or decelerate in a very short time (almost abruptly). Moreover, not only the economic growth can slow down, but also the growth of science, culture, and so on.

Thus, nearby to the moment of exacerbation an abrupt acceleration of the development processes as well as their equally sharp slowdown can take place. The competition of two factors (slowdown and acceleration) in the nonlinear system causes this situation. We consider the dynamics of the socio-economic systems development in the open nonlinear medium based on two groups of factors. On the one hand, these factors create the so-called heterogeneity in the nonlinear media. In general, this is the effect of nonlinear feedbacks that contributes self-increasing (or self-extenuation) of the processes in the socio-economic system. On the other hand, these are factors eroding inhomogeneities in the nonlinear media, what is an analogue of the dissipation process. Scattering factors "work" through the influence mechanisms processes occurring at the micro economic level as well as the development processes at the macro level.

Let's return to the situation on Moscow stock-exchange on the 30th and 31st October in 2014, when the abnormal leaps of ruble rate, as a result of which the Russian currency strengthened averagely for $4 \%$ against dollar and euro, and on the next day the ruble lost $3 \%$.

The experts consider that the sharp leaps of ruble rate are conditioned by the game of speculators on the currency market, as on the 31 st October the strengthening of the ruble after increase of key rate from $8 \%$ to straight away 9.5\% by the Central Bank of Russia was expected, but the effect turned out to be reverse.

The beginning of November 2014 was marked by that the index of RTS for the first time for five years dropped lower than 1000 points. Based on the background of the Russian stock market the national currency of Russia 
daily reduced almost for 3\%. Euro for the first time overcame the mark in 60 RUB, followed by renovation of the historical minimum by the dollar, when first rose over 48 RUB, at that the demand on foreign currency on the part of population has grown in 3-4 times in comparison to the beginning of the year.

The financiers are frightened that such fluctuations of the ruble rate take place on the threshold of Centrobank solution about transition on the "floating" ruble, as then volatility on the market can considerably grow up, and it is inappropriate for the economy of the country, if the rate of national currency would regain and then lose for several per cents a day. However, simultaneously the panic among population that can enhance the capital outflow from the country can become the destabilizing factor.

The contest between these groups of factors such as the nonlinear positive feedbacks influence and dissipative scattering processes leads to different modes of the development processes in socio-economic systems. From one point of view, the localization, the intensity growth and the processes productivity mode in a rather narrow field next to the aggravation can be established if the nonlinear positive feedbacks are stronger than the dissipation analogue. From another one there is a possibility that the economic processes intensity reduction, energy and structures dissipation and their "spreading from the center" mode plays more important role if the dissipation analogue turns out to be stronger than the self-stimulating development factor.

Taking all the aforesaid into consideration, we can conclude that the development of complex socio-economic systems is performed with the saturation as it approaches its parameters to some naturally formed limit. Such a state in the economic systems is usually called stagnant, when the system spends all the resources and capabilities to duplicate itself and its efficiency, size, internal content and so on. The economics having reached this level not only can reduce the consumption of resources, but they also objectively are obliged to increase the flow of almost all the types of resources, what is necessary to replace technologically and technically obsoleting means of production, rising costs of energy, information, materials needed to maintain the attained level of the development. Therefore, to manage effectively the economic development in the state of stagnation, recession, etc., to form relevant structure-attractors, "attracting" favorable development trajectories, there is a requirement to create a new management paradigm, based on the idea of economic evolution and synergy, considering the basic principles of evolution and self-organization.

Thus, the fundamental principle of the complex socio-economic systems development is the periodic alternation of attenuation and amplification stages, activity explosions and damping, integration and disintegration, and decay. From the synergetic standpoint, in order to achieve sustainable development of such systems it is required not only a certain degree of chaos (like natural factors, market-based mechanisms of selection and survival of the strongest ones), spontaneity of evolvement and self-management, but also the effective external control, which assumes surveillance and coordination of the system certain components. Except that both of these components should be balanced and coordinated with each other.

\section{Conclusions}

The analysis of theoreticals and practice of the economic systems regulation in periods of instability, economical crisis or stagnation leads to the conclusion: the insufficient success to realize strategic goals and the lack of progress to overcome the adverse trends prevailing in the economy are in a great measure conditioned by the following reasons:

1) Ongoing appearance of both ultra small and significant changes (fluctuations) in the external and internal environment of the various economic systems what determines their transition from a relatively stable to an unstable evolutionary state;

2) The fundamental impossibility to realize certain ambitious strategic aims as a consequence of insufficient accounting of positive and negative feedbacks effect in the economy (in case of the inadequate strategy elaboration desired goals can not be achieved);

3) The lack of means and effective methods of economics management the during periods of extreme volatility, especially in the peak moment.

Nowadays, due to the deficiency of the holistic concept and scientifically based recommendations how to manage economic systems in periods of the extreme instability, stagnation and recession, the socio-economic development and transformation of the Russian economy proceeds in a complicated and contradictory way.

Yet to be solved remain the contradiction between the existing practice of economic development and the management of complex economic dynamics in the Russian Federation, as well as those mechanisms of state regulations that are meant to ensure effective development and transformation of economic systems in a predetermined (preset) direction. 
The formation of prerequisites to transit the Russian economy to the innovative development, overcome the adverse trends in periods of the high economic instability is associated with the development of new theoretical and methodological approaches to the analysis and the forecasting economic systems development and the creation of effective mechanisms and means to control them.

The self-organization concept as well as the economic evolution theory which rapidly develops allow to answer the crucial question how to build a modern management system combining the focus on economic development, perfect functions execution, security, and relations of democratic nature and the creative spirit giving a birth to ideas and innovations with no limit.

Economics as a nonlinear evolving system is affected by the state. The reaction of the economy to its decisions and acts is often unexpected that the state's specificity determines as a complex structural system. Challenges of globalization and the necessity of innovative development give rise to the critical issue imperatively requiring to constitute a new type of state administration capable of adequate, punctual and competent respond to the already launched demands as well as anticipating and preventing of potential ones.

Consequently, the ability of state institutions to develop and implement a policy of social and economic changes taking into the consideration the evolutionary cyclical dynamics of economy and society development defines the quality of governance in contemporary conditions. This is necessary but not to impose the outlying or unrealizable development vectors and trajectories that will have a deterrent effect on innovative development of the country and ensuring its competitiveness and economic security.

Today's crisis and sharp weakening of the ruble is caused by the shock after the Central Bank radically changed the rate and supervision policy. It is expected that when the shock is over, the ruble will begin restoration. The Russian currency must be supported by REPO currency from the Centrobank that would be the stabilizing factor.

In these conditions a lot depends on the further policy of Centrobank, where reduction of inflation expectations is now the priority. The market volatility remains high enough on the background of ambiguity, multiply-factor nature and heavily prognosticated tendencies, connected with high geopolitical risks and drop of the world prices on the oil. The policy of a regulator on this stage must be especially weighed (braking and acceleration) in the financial-economic system, as well as action of the non-linear feedbacks, which promote to self-growth (or self-weakening) of the positive (or negative) processes in the social-economic system.

To summarize let's mark that we are forced to acknowledge that the existing Russian model of the financial regulation and supervision doesn't provide the function imposed on it in relation to provision of the financial-economic system stability in the conditions of grown geopolitical risks and situation in the world economy on the whole. The practice showed that such model in its essence is not able to execute this task, as it is oriented on the provision of stability and reliability of only separate elements on the national financial system. The increase of system stability on the whole can be provided through implementation of the effective state financial-economic policy, supposing the creation of the new model of regulation, based upon usage of the strict criteria, indicators, instruments, which allow detecting and taking the preventive measures on prevention of the destabilizing processes, and implementing the measures on strengthening of the financial sector stability and economy of the country on the whole in a proper time.

\section{References}

Afonasova, M. A. (2009). Innovative modernization of the Russian economy: A regional perspective. Tomsk: State University of Control Systems and Radio electronics.

Afonasova, M. A. (2010). Management of Innovative Development of the Region in Terms of Institutional Transformation of the Economy. Saint Petersburg: Litera.

Bransky, V. P. (2004). Self-Organization and Management. In Strategy of the dynamic development of Russia: The unity of self-organization and management. Moscow: Nauka.

Ebeling, V., \& Feistel, R. (2005). Chaos and Cosmos: Evolution Synergetic. Moscow, Izhevsk: Institute of Computer Studies.

Friedman, M. R. (1980). Free to Choose. A Personal Statement. NY: Liberty Publishing House.

Knyazeva, E. N. (2005). Kurdumov S. P. Synergetic paradigm. Basic concepts in the context of the history of culture. Retrieved from http://www.lib.icr.su/node/1247

Malinetsky, G. G. (2006). Future of Russia in the Mirror of Synergy. Moscov: KomKniga.

Moiseyev, N. N. (1999). The Logic of Dynamic Systems and the Development of Nature and Society. Problems 
of Philosophy, 4, 5-11.

Nicolis, G., \& Prigogine I. (1979). Self-organization in non-equilibrium systems. From dissipative structures to order in terms of fluctuations. Moscow: Nauka.

Nicolis, G., \& Prigogine, I. (1990). Exploring of Complexity. Moscow: Nauka.

\section{Copyrights}

Copyright for this article is retained by the authors, with first publication rights granted to the journal.

This is an open-access article distributed under the terms and conditions of the Creative Commons Attribution license (http://creativecommons.org/licenses/by/3.0/). 